\title{
CHARCOT-MARIE-TOOTH AND ANALGESIA FOR LABOR: CASE REPORT
}

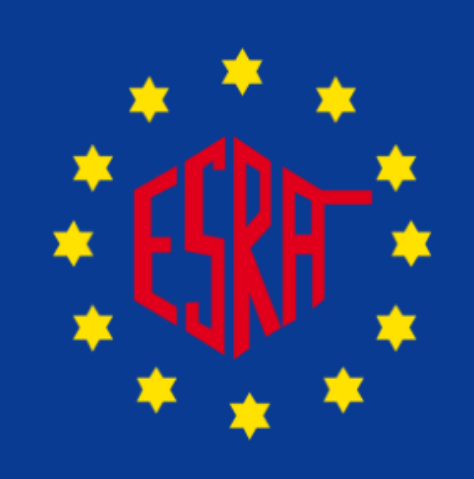

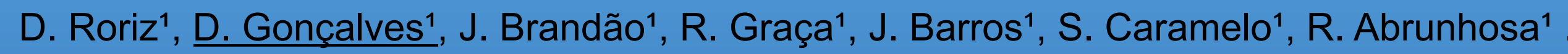
${ }^{1}$ Centro Hospitalar de Trás-os-Montes e Alto Douro, Anesthesiology and Pain Therapy, Vila Real (Portugal).

Background: Charcot-Marie-Tooth disease (CMT) is a demyelinating polyneuropathy, of hereditary character, caused by defects in myelin structure, maintenance and formation. This can lead to progressive distal muscle weakness and wasting as well as sensory loss, which is often coupled with neuropathic pain. Recent research reveals a wide variability of genotypic and phenotypic subgroups, some of which, they have important anesthetic implications. We present a case of presumable CMT that underwent epidural analgesia during labor and delivery.

Methods: A 37-year-old woman, ASA III, at approximately 40 weeks' gestation, was admitted to the emergency unit in labor. The patient had a family history of CMT (father and cousin), but herself without a diagnostic of the disease (genetic test). During physical examination, we noticed bone deformity in the left foot, decreased muscle strength, numbness and tingling in the lower limbs, which worsened during pregnancy.

\section{(continuous methods)}

After she expressed desire for a regional pain management, she was informed about the risks of performing the neuroaxis approach and has accepted the technique. Given the possibility of CMT disease, we opted for epidural analgesia. The epidural technique was performed with an 18G Tuohy needle at the L3-L4 level, leaving $4 \mathrm{~cm}$ of catheter in the epidural space.

The technique went on without complications.

Results: In the 6 following hours there was no motor blockage of any grade and T8 sensitive blockade was established, providing effective analgesia. The vaginal delivery was not instrumented and went on without complications. There was complete recovery of the sensory block after two hours.

The patient was discharged 2 days after delivery, without any complications.

Conclusion: Taking into account that the literature on the epidural technique for labor analgesia in a patient with CMT is scarce, we think it could represent a safe option and alternative; especially considering the main causes of maternal morbidity and mortality, like failure in orotracheal intubation and higher probability of bronchoaspiration, associated in particular with general anesthesia, if anesthesia is necessary.

A. Pérez, C.García, O. González, J. Estévez. Charcot-Marie-Tooth y analgesia epidural obstétrica, 